\title{
Highlights from LHCb
}

\author{
Albert Bursche ${ }^{1,2, \star}$ on behalf of the LHCb Collaboration \\ ${ }^{1}$ Università degli studi di Cagliari, Italy \\ ${ }^{2}$ INFN Cagliari, Italy
}

\begin{abstract}
The recent highlights from LHCb in soft QCD and Heavy Ion physics are presented. This includes measurements from collisions of proton and lead ${ }_{82}^{208} \mathrm{~Pb}^{82+}$ ion beams with other beams as well as noble gas targets. An outlook on future analyses of ${ }_{54}^{129} \mathrm{Xe}^{54+}$ collisions is presented.
\end{abstract}

\section{Introduction}

The LHCb detector is a unique apparatus for soft QCD and Heavy Ion physics. With the forward acceptance at high rapidity $(y) 2<y<5$; with excellent tracking; particle identification capabilities and a system for noble gas injection - the SMOG system - for fixed target collisions [1] with the LHC beam LHCb can do measurements no other experiment can do. Additional forward scintillators (HeRSCheL) in the region $5<\eta<9$ can be used to select central exclusive events.

\section{Fixed Target Physics}

For fixed target physics in LHCb the SMOG system is used [1]. This system allows for the injection of noble gases in the accelerator vacuum. LHCb's acceptance then corresponds to a central rapidity range. LHCb has recorded the collisions of protons with Helium; Neon and Argon as well as collisions of lead ${ }_{82}^{208} \mathrm{~Pb}^{82+}$ ions with Argon.

\subsection{Antiproton production in proton helium collisions at $\sqrt{s_{N N}}=110 \mathrm{GeV}$}

Antiproton production in proton helium collisions is currently not well understood and is an important ingredient to the transport models of high energy cosmic rays $[2,3]$. The double differential cross section of antiproton production has been measured as a function of antiproton momentum and transverse momentum [4]; compared to EPOS LHC [5] and is presented in figure 1. This result is currently being updated with an improved determination of the luminosity for publication.

\subsection{Open Charm and Charmonium Production in Proton Argon Collisions at $\sqrt{s_{N N}}=110 \mathrm{GeV}$}

Production of $J / \psi$ and $D^{0}$ mesons is observed in proton Argon collisions at $\sqrt{s_{N N}}=110 \mathrm{GeV}$. Cross Section and yields are measured differentially in transverse momentum and rapidity [8]. There is evidence for $D^{+}, D^{*+}, D_{s}^{+}$and $\Lambda_{c}^{+}$production.

^e-mail: albert.bursche@cern.ch 


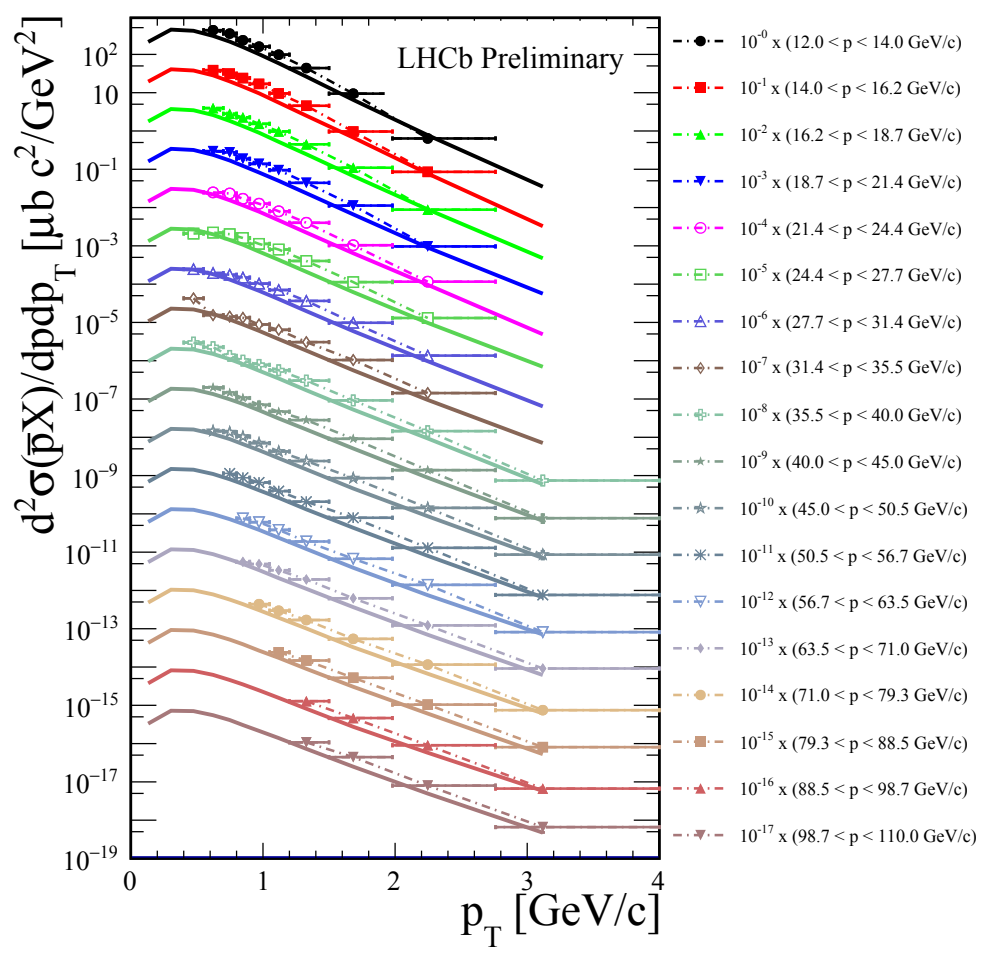

Figure 1. Antiproton production cross section in pHe collisions[4] measured double differentially in momentum and transverse momentum. Compared to EPOS LHC absolute predictions [5]. EPOS LHC predictions are systematically underestimating the data by a factor 1.5. Better agreement is found with EPOS 1.99 and HIJING.

\section{Collider Mode}

\subsection{First Observation of $\Xi_{c c}^{++}$}

The first observation of the double charmed baryon $\Xi_{c c}^{++}$with the mass of

$$
m_{\Xi_{c c}^{++}}^{+\infty}=3621.40 \pm 0.72 \pm 0.27 \pm 0.140 \mathrm{MeV} / c^{2}
$$

is reported [6]. The first uncertainty is statistical; the second is systematic and the third originates from the limited knowledge of the $\Lambda_{c}$ mass. This measurement was performed using a data set corresponding to $2 \mathrm{fb}^{-1}$ at $\sqrt{s}=8 \mathrm{TeV}$ and checked using $1 \mathrm{fb}^{-1}$ at $\sqrt{s}=7 \mathrm{TeV}$ of proton proton collisions. This observation is in tension with a $\Xi_{c c}^{+}$state reported by the SELEX collaboration [7]. The difference to the mass reported by SELEX,

$$
m_{\Xi_{c c}^{+}}=3519 \pm 2 \mathrm{MeV} / c^{2}
$$

is inconsistent with the presumption that those two states are separated by isospin only.

\subsection{Open Charm Production at $\sqrt{s}=5 \mathrm{TeV}$ in Proton Proton Collisions}

Measurements of the double differential cross section for $D^{0}, D^{+}, D_{s}^{+}$and $D^{+*}$ production and the production ratios between $13 \mathrm{TeV}$ and $5 \mathrm{TeV}$ have been reported [9]. The data generally agree well 
with the FONLL, POWHEG+NNPDF3.0L and GMVFNS predictions for the ratios of cross-sections at $\sqrt{s}=13 \mathrm{TeV}$ and $\sqrt{s}=5 \mathrm{TeV}$.

\subsection{Open Charm Production in Collisions of ${ }_{82}^{208} \mathrm{~Pb}^{82+}$ Ions and Protons at $\sqrt{s_{N N}}=5 \mathrm{TeV}$}

Prompt $D^{0}$ production was measured in proton ${ }_{82}^{208} \mathrm{~Pb}^{82+}$ collisions. Double differential cross sections $\left(y, p_{T}\right)$; forward backward ratios and nuclear modification factors have been measured [10]. The nuclear modification factor is shown in figure 2 .

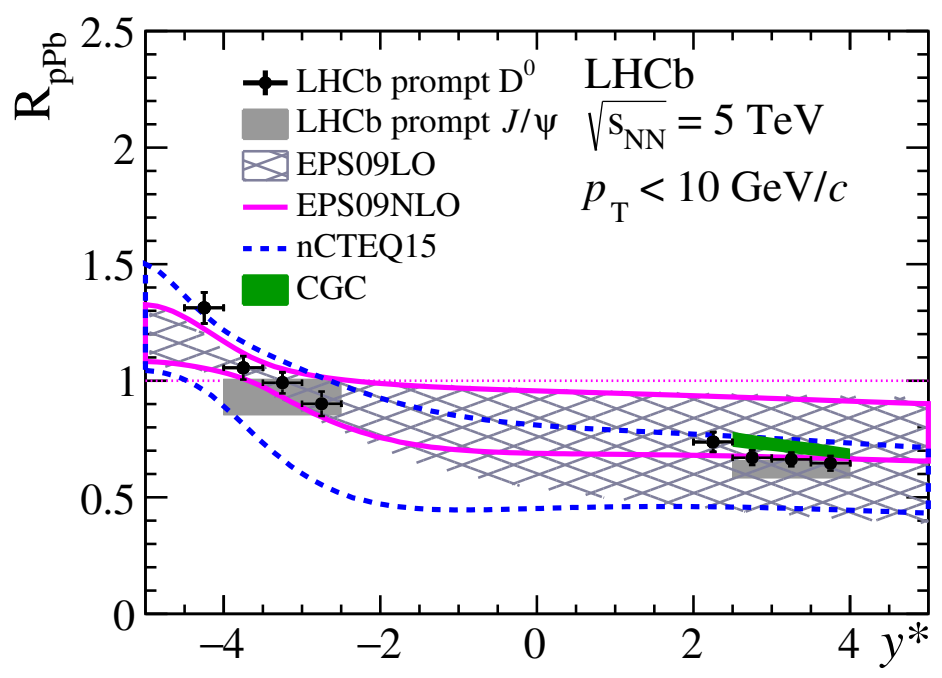

Figure 2. Nuclear modification factor for $D^{0}$ production as a function of the rapidity in the centre of mass frame $\left(y^{*}\right)$ at $\sqrt{s_{N N}}=5 \mathrm{TeV}$ compared to predictions using nuclear PDFs (EPS09, EPS09NLO, nCTEQ15)[11-13] and a colour glass condensate model (CGC) [14].

\subsection{Charmonium Production in Collisions of ${ }_{82}^{208} \mathrm{~Pb}^{82+}$ Ions and Protons at $\sqrt{s_{N N}}=8.16 \mathrm{TeV}$}

Charmonium production in collisions of ${ }_{82}^{208} \mathrm{~Pb}^{82+}$ ions and protons at $\sqrt{s_{N N}}=8.16 \mathrm{TeV}$ has been measured. The prompt component and the $J / \psi$ component originating from beauty quark decays are separated in this measurement. Nuclear modification factor and forward backward ratio have been measured. The nuclear modification factor is shown in figure 3.

There is evidence for $D^{+}, D_{s}^{+}, \Lambda_{c}^{+}$production as well as exclusive $\rho^{0}$ production in ultraperipheral collisions.

\subsection{Collisions of ${ }_{82}^{208} \mathrm{~Pb}^{82+}$ lons at $\sqrt{s_{N N}}=5 \mathrm{TeV}$}

The analyses of the data collected in ${ }_{82}^{208} \mathrm{~Pb}^{82+}$ collisions are still ongoing. Evidence for open charm, open strange and charmonium production in ion collisions in both mesonic and barionic final states up to $50 \%$ centrality was presented. There is also clear evidence of exclusive charmonium production in ultraperiphereal collisions which is currently under study. 

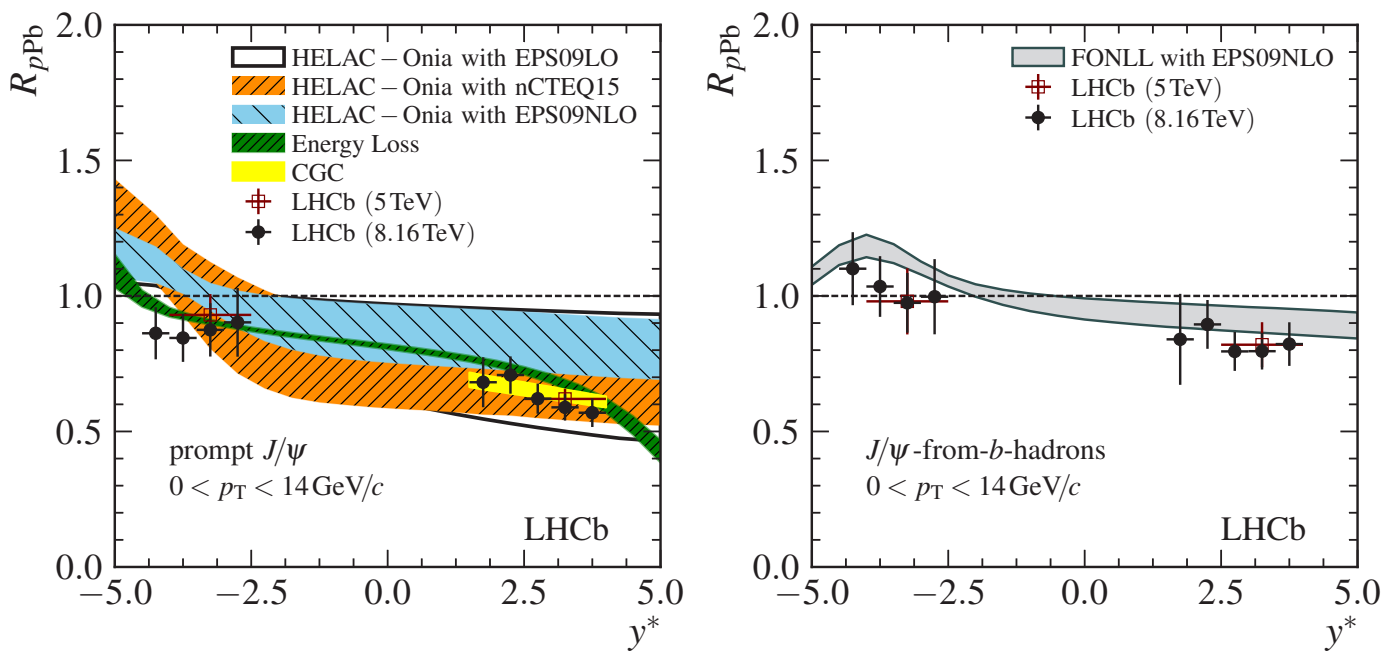

Figure 3. Nuclear modification factor as a function of the rapidity in the centre of mass frame $\left(y^{*}\right)$ for prompt (left) and non-prompt (right) $J / \psi$ production at $\sqrt{s_{N N}}=5 \mathrm{TeV}$ and at $\sqrt{s_{N N}}=8.16 \mathrm{TeV}$ compared to predictions from HELAC-Onia [11, 12] using EPS09LO [15]; nCTEQ15 [16] and EPS09NLO [15] nuclear parton distribution functions and GCC and an energy loss model in the prompt case and to FONLL [17, 18] using EPS09NLO [15] in the detached case.

\section{Conclusions}

The efforts to analyse ion collisions in LHCb start to bear fruits. Despite being geared for heavy flavour production in proton collisions and missing out on the first lead run of the LHC the LHCb collaboration is gaining momentum in heavy ion physics. Especially in the asymmetric collisions of protons with lead more interesting results are in preparation. The recently finished LHC run with xenon beams confirmed the LHCb capability to observe heavy flavour production in heavy ion collisions. Evidence for charm production shown in figure 4.
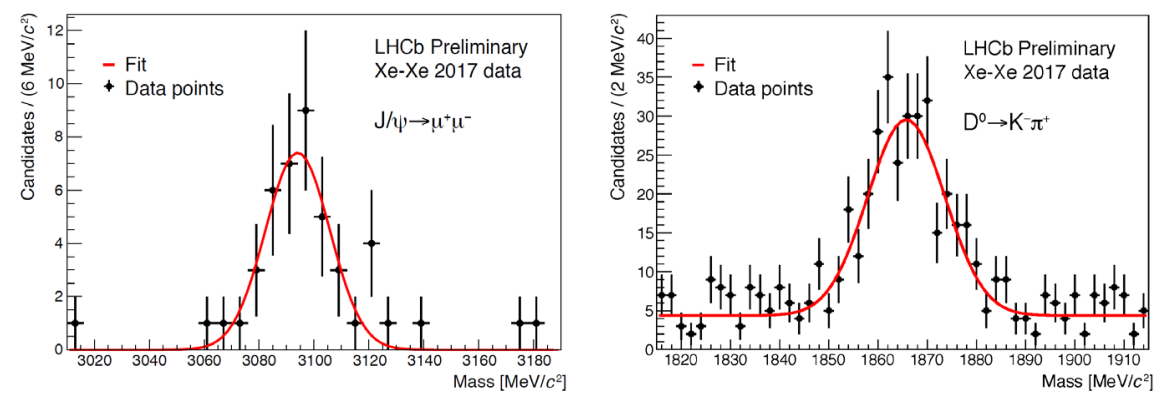

Figure 4. Invariant mass of dimuons and $K^{ \pm} \pi^{\mp}$ measured in $\left({ }_{54}^{129} \mathrm{Xe}^{54+}\right)$ collisions at $\sqrt{s_{N N}}=5.44 \mathrm{TeV}$. 


\section{References}

[1] LHCb Collaboration JINST 9 P12005 (2014)

[2] PAMELA Collaboration JETP Letters 96621 (2013)

[3] AMS Collaboration Phys. Rev. Lett. 117091103 (2016)

[4] LHCb Collaboration preliminary LHCb-CONF-2017-002

[5] T. Pierog et. al. Phys. Rev. C95 034906 (2015)

[6] LHCb Collaboration Phys. Rev. Lett. 119112001 (2017)

[7] SELEX Collaboration Phys. Rev. Lett. 89112001 (2002)

[8] LHCb Collaboration preliminary LHCb-CONF-2017-001 (2017)

[9] LHCb Collaboration JHEP 06147 (2017)

[10] LHCb Collaboration JHEP 10090 (2017)

[11] J.-P. Lansberg and H.-S. Shao Eur. Phys. J. C77 1 (2017)

[12] H.-S. Shao Comput. Phys. Commun. 1842562 (2013)

[13] H.-S. Shao Comput. Phys. Commun. 189238 (2016)

[14] B. Ducloué, T. Lappi, and H. Mäntysaari Phys Rev. D91 114005 (2015)

[15] K. J. Eskola, H. Paukkunen, and C. A. Salgado JHEP 04065 (2009)

[16] K. Kovarik et. al. Phys. Rev. D93 085037 (2016)

[17] M. Cacciari, M. Greco, and P. Nason JHEP 05007 (1998)

[18] M. Cacciari, M. Greco, and P. Nason JHEP 03006 (2001) 
